\title{
Optimization design of heat pipe heat exchanger using response surface methodology
}

\author{
Shuai $\mathrm{Pu}^{1 *}$, Wei Huang ${ }^{1}$ \\ ${ }^{1}$ Nuclear Power Institute of China, Chengdu, Sichuan 610200, China
}

\begin{abstract}
In this paper, Optimization design of heat pipe heat exchanger (HPHX) is processed utilizing the Response Surface Methodology (RSM). The response surface model was built by regressive analysis using Latin hypercube experimental design method and numerical simulation. Through response surface analysis, it is found that the two input variables affecting the performance of HPHX are the heat pipe pitch and the Inlet and outlet distance. Moreover, the maximum value of the overall performance factor on the response surface is searched using genetic algorithm, and the optimal values of four input variables are obtained.
\end{abstract}

\section{Introduction}

HPHXs are widely used in industrial processes due to their excellent performance, and the design of HPHX is of great importance. The main objective of HPHX design is to achieve higher overall performance. In this paper, combined with numerical simulation, RSM is utilized to perform the optimization design of the HPHX.

\section{Response surface methodology}

The basic idea of RSM is to use simple polynomial model to describe the real complex model approximately in a certain value range [1]. Following steps are considered in RSM:

1. Generating a certain number of sample points in the value range of input variables by a reasonable experimental design method.

2. Calculating the values of corresponding output variables by numerical calculation.

3. Establishing an approximate model (response surface) between the input variables and the output variables using polynomial model.

4. Searching the global optimization point using optimization algorithms.

\subsection{Latin hypercube experimental design method}

In order to ensure that the fitted response surface is a reasonable approximation of the actual problem, it is necessary to properly use the experimental design method to generate a set of sample points, on the basis of which the response surface can be efficiently established. This paper will adopt Latin hypercube experimental design method.

Assuming that the output variable $y$ is a deterministic function of the input variable $x_{i}(i=1,2,3 \ldots k)$, the basic idea of Latin hypercube sampling can be summarized as follows [2]:

(1)Assuming that $\mathrm{m}$ samples are needed, the value range of input variable $x_{i}$ is divided into $\mathrm{m}$ equal probability intervals, as shown in the figure, sample values are randomly generated in each interval, and $\mathrm{m}$ sample values are obtained for each input variable $x_{i}$, which is denoted as $x_{i 1}, x_{i 2}, x_{i 3}, \ldots, x_{i m}(i=1,2,3 \ldots k)$.

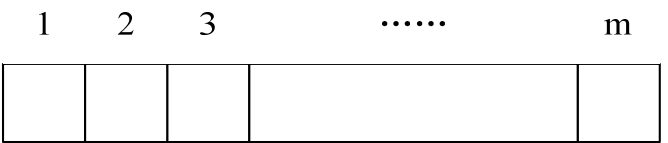

Fig 1 Division of Latin hypercube sampling interval.

(2)Randomly combining $x_{11}, x_{12}, x_{13}, \ldots, x_{1 m}$ and $x_{21}, x_{22}, x_{23}, \ldots, x_{2 m}$ to obtain $\mathrm{m}$ groups of data, which can be recorded as $\mathrm{m} \times 2$ matrix, and then randomly combining these $\mathrm{m}$ groups of data with $x_{31}, x_{32}, x_{33}, \ldots, x_{3 m}$ to obtain $\mathrm{m} \times 3$ matrix, ..., until randomly combining with $x_{k 1}, x_{k 2}, x_{k 3}, \ldots, x_{k m}$ to obtain $\mathrm{m} \times \mathrm{k}$ matrix.

$$
\left[\begin{array}{cc}
x_{1 a} & x_{2 \mathrm{o}} \\
x_{1 b} & x_{2 p} \\
\vdots & \vdots \\
x_{1 c} & x_{2 q}
\end{array}\right]
$$




$$
\boldsymbol{x}=\left[\begin{array}{cccc}
x_{1 a} & x_{20} & \cdots & x_{k r} \\
x_{1 b} & x_{2 p} & \cdots & x_{k s} \\
\vdots & \vdots & & \vdots \\
x_{1 c} & x_{2 q} & \cdots & x_{k t}
\end{array}\right]
$$

$$
a, b, c, o, p, q, r, s, t=1,2, \ldots, m \text {. }
$$

The $\mathrm{m}$ rows in matrix (2) represents $\mathrm{m}$ sample points obtained by Latin hypercube sampling, which are denoted as $\boldsymbol{x}^{(1)}, \ldots, \boldsymbol{x}^{(\mathrm{m})}$, and $\mathrm{k}$ values in each row are the values of each input variable.

(3) Using $\mathrm{m}$ sample points, the output variable $y_{j}(j=1,2, \ldots, m)$ is calculated and recorded as matrix $\boldsymbol{y}=\left[y_{1}, y_{2}, \ldots, y_{m}\right]^{\mathrm{T}}$.

Latin hypercube sampling can be regarded as a compromise process, which combines many desirable characteristics of random sampling and stratified sampling.

\subsection{Response surface model}

The RSM replaces the actual complex model with a simple polynomial function. When the range of input variables is small, the lower-order polynomial model can get a better approximation effect. The lower-order polynomial model can be written in the following form:

$$
y\left(x_{1}, x_{2}, \ldots, x_{k}\right)=\beta_{0}+\sum_{i=1}^{N} \beta_{i} \varphi_{i}(\boldsymbol{x})
$$

$\varphi_{i}(x)$ is the basis function, $N$ is the number of basis functions, the number of basis functions of firstorder and second-order polynomial models are $N=k$ and $N=C_{k}^{l}+C_{k}^{l}+C_{k}^{2}=C_{k+2}^{2}-1$ respectively, and $\beta_{i}$ is the basis function coefficient. The regression coefficient $\boldsymbol{\beta}=\left(\beta_{0}, \beta_{1}, \ldots, \beta_{N}\right)^{T}$ is obtained by the least square method [3]:

$$
\begin{aligned}
\boldsymbol{\beta} & =\left(\boldsymbol{\phi}^{T} \boldsymbol{\phi}\right)^{-1}\left(\boldsymbol{\phi}^{T} \boldsymbol{y}\right) \\
\boldsymbol{\phi} & =\left[\begin{array}{ccccc}
1 & \varphi_{1}\left(\boldsymbol{x}^{(1)}\right) & \varphi_{2}\left(\boldsymbol{x}^{(1)}\right) & \ldots & \varphi_{\mathrm{N}}\left(\boldsymbol{x}^{(1)}\right) \\
1 & \varphi_{1}\left(\boldsymbol{x}^{(2)}\right) & \varphi_{2}\left(\boldsymbol{x}^{(2)}\right) & \cdots & \varphi_{\mathrm{N}}\left(\boldsymbol{x}^{(2)}\right) \\
\vdots & \vdots & \vdots & & \vdots \\
1 & \varphi_{1}\left(\boldsymbol{x}^{(\mathrm{m})}\right) & \varphi_{2}\left(\boldsymbol{x}^{(\mathrm{m})}\right) & \cdots & \varphi_{\mathrm{N}}\left(\boldsymbol{x}^{(\mathrm{m})}\right)
\end{array}\right]
\end{aligned}
$$

\section{Optimization procedure}

Figure 2 shows a HPHX using 19 heat pipes as heat transfer elements. The performance of the HPHX is affected by many parameters. This paper utilizes RSM to perform the optimization design of the HPHX.

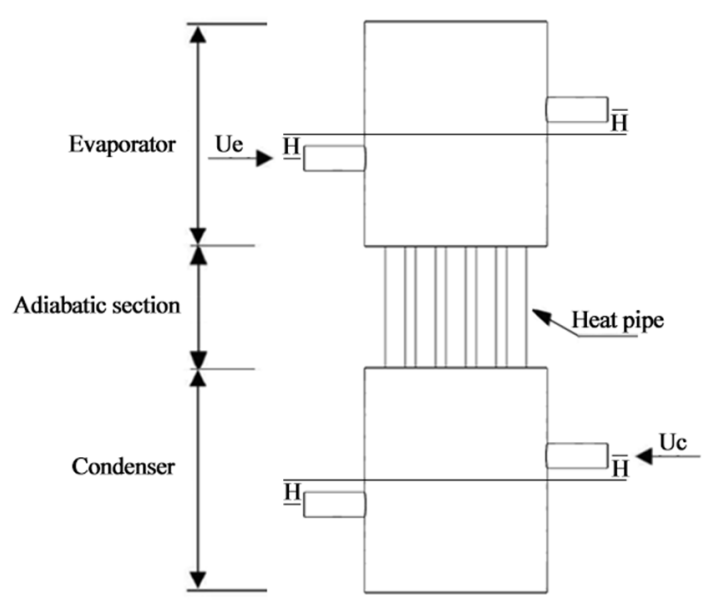

(a)

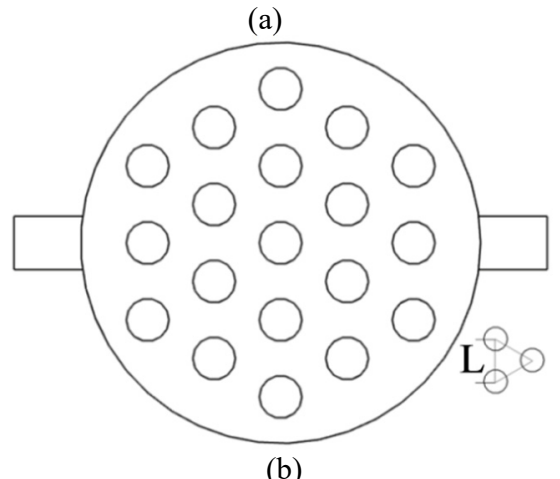

Fig. 2 .HPHX sketch (a) and heat pipe arrangement (b).

\subsection{Numerical model}

The heat pipes are assumed as solid thermal conductors with a constant thermal conductivity using thermal resistance model [4]. Velocity inlet and pressure outlet are applied to the inlets and outlets respectively. No-slip wall boundary conditions are applied to all wall surfaces.

\subsection{Input variables}

Four input variables are shown in figure 2. And their value range is shown in table 1 .

Table 1. Input variables and value range.

\begin{tabular}{|c|c|c|}
\hline Input variables & Symbol (Unit) & Value range \\
\hline Heat pipe pitch & $\mathrm{L}(\mathrm{mm})$ & $24-28.8$ \\
\hline Inlet and outlet distance & $\mathrm{H}(\mathrm{mm})$ & $0-70$ \\
\hline Inlet velocity of evaporator & $\mathrm{Ue}(\mathrm{m} / \mathrm{s})$ & $1.326-2.55$ \\
\hline Inlet velocity of condenser & $\mathrm{Uc}(\mathrm{m} / \mathrm{s})$ & $6.63-14.7$ \\
\hline
\end{tabular}

\subsection{Objective function}

The overall performance factor $(\eta)$ of a heat exchanger is defined as follows [5]:

$$
\eta=\frac{N u}{\xi^{1 / 3}}
$$

where $\xi$ is the dimensionless drag coefficient expressed as follows:

$$
\xi=\frac{\Delta P}{\rho \cdot u^{2} / 2}
$$

The larger the $\eta$, the better the overall performance of 
the heat exchanger. Thus this paper takes $\eta$ as the optimization goal:

$$
\max F=\eta(L, H, U e, U c)
$$

\section{Results and discussion}

\subsection{Results of the experiments}

25 sample points are generated using Latin hypercube sampling, and the numerical simulations of 25 runs are performed to obtain the output variable $\eta$. The sample points and results are shown in Table 2.

Table 2. The results of the experiments for $\eta$.

\begin{tabular}{|c|c|c|c|c|c|}
\hline $\mathrm{S} / \mathrm{N}$ & $\mathrm{L}(\mathrm{mm})$ & $\mathrm{H}(\mathrm{mm})$ & $\mathrm{Ue}(\mathrm{m} / \mathrm{s})$ & $\mathrm{Uc}(\mathrm{m} / \mathrm{s})$ & $\eta$ \\
\hline 1 & 26.4 & 32.2 & 1.8 & 7.1 & 46.9 \\
\hline 2 & 27.2 & 29.4 & 2.2 & 12 & 42.7 \\
\hline 3 & 24.1 & 12.6 & 1.9 & 13.2 & 35 \\
\hline 4 & 26.8 & 26.6 & 2.5 & 11 & 42.2 \\
\hline 5 & 24.5 & 54.6 & 1.5 & 8.7 & 45.8 \\
\hline 6 & 28.5 & 60.2 & 2.1 & 8.4 & 57.1 \\
\hline 7 & 26.6 & 57.4 & 2.2 & 12.3 & 52.8 \\
\hline 8 & 28.3 & 35 & 1.6 & 11.3 & 45.2 \\
\hline 9 & 25.4 & 49 & 2.3 & 9.1 & 46.5 \\
\hline 10 & 25.1 & 68.6 & 1.6 & 14.5 & 47.3 \\
\hline 11 & 25.8 & 4.2 & 1.4 & 6.8 & 33.1 \\
\hline 12 & 24.9 & 37.8 & 2.4 & 10.3 & 41.6 \\
\hline 13 & 26.2 & 21 & 2.4 & 9.4 & 39.1 \\
\hline 14 & 27.4 & 7 & 1.8 & 13.6 & 30.7 \\
\hline 15 & 25.6 & 63 & 2.5 & 8.1 & 49.1 \\
\hline 16 & 27.9 & 23.8 & 1.7 & 12.6 & 42.2 \\
\hline 17 & 27.7 & 65.8 & 1.7 & 10 & 58.1 \\
\hline 18 & 27 & 9.8 & 2 & 13.9 & 31.9 \\
\hline 19 & 24.7 & 15.4 & 1.4 & 9.7 & 37.3 \\
\hline 20 & 24.3 & 18.2 & 2.1 & 14.2 & 34.7 \\
\hline 21 & 26 & 51.8 & 2 & 11.6 & 47.4 \\
\hline 22 & 28.1 & 1.4 & 2.3 & 7.4 & 33.2 \\
\hline 23 & 28.7 & 40.6 & 1.9 & 12.9 & 50.2 \\
\hline 24 & 25.2 & 46.2 & 1.5 & 7.8 & 44.3 \\
\hline 25 & 27.6 & 43.4 & 1.4 & 10.7 & 52.6 \\
\hline
\end{tabular}

\subsection{Discussion}

Apply the least squares method to the data in Table 2 to get the regression equation of the response surface. The regression equation is as follows. A, B, C, D refers to L, $\mathrm{Ue}, \mathrm{Uc}, \mathrm{H}$ respectively.

$\eta=-73.0091+7.64186 \times \mathrm{A}-3.05984 \times \mathrm{B}$

$+3.30378 \times \mathrm{C}-1.25715 \times \mathrm{D}+0.18683 \times \mathrm{AB}$

$-0.087811 \times \mathrm{AC}+0.065683 \times \mathrm{AD}-0.35396 \times \mathrm{BC}$

$-0.025123 \times \mathrm{BD}+9.09269 \times 10^{-3} \times \mathrm{CD}-0.14599 \times \mathrm{A}^{2}$

$+0.45733 \times \mathrm{B}^{2}-0.046844 \times \mathrm{C}^{2}-2.80748 \times 10^{-3} \times \mathrm{D}^{2}$

Figure 3 shows the residual plots of the regression equation. The residual satisfies the homogeneity of variance and obey the normal probability distribution. Thus the regression equation is reliable.

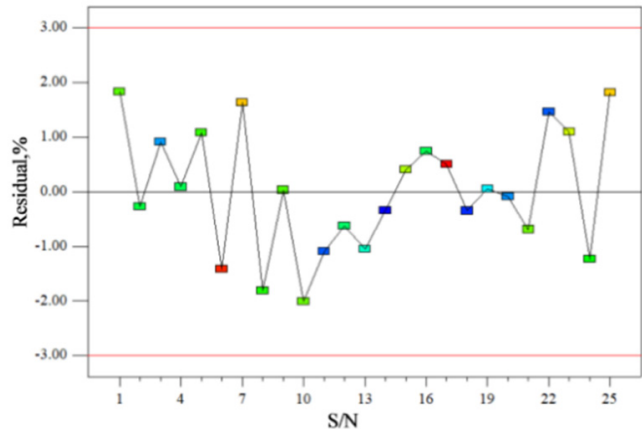

(a) Residual vs. S/N.

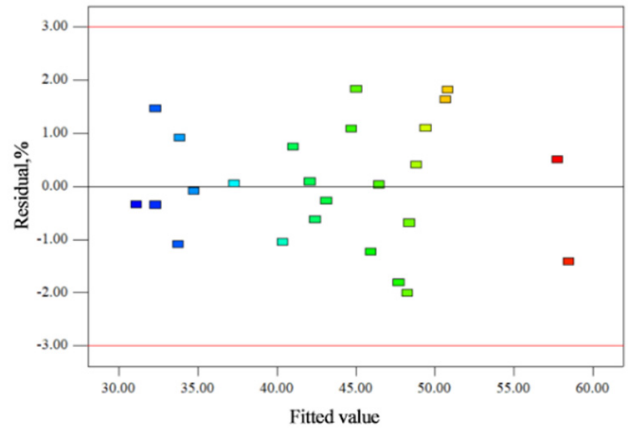

(b) Residual vs. fitted value.

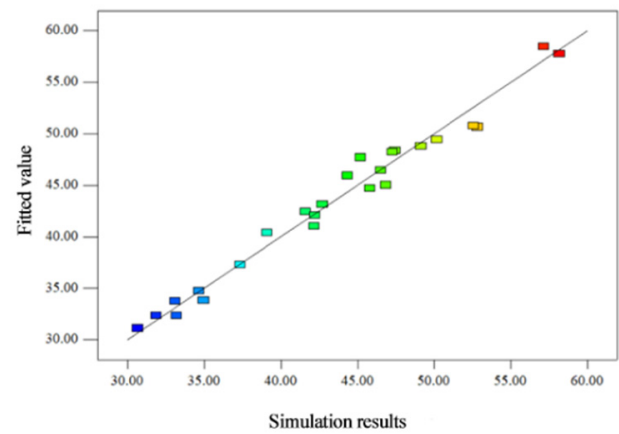

(c) Fitted value vs. simulation results.

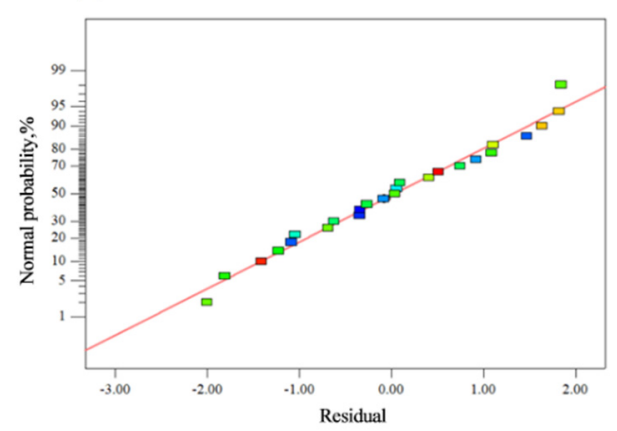

(d) Normal probability plot.

Fig. 3 .Residual plots of $\eta$ (a, b, c, d).

\subsubsection{Response surface}

Figure 4 shows the response surface of $\eta$ with respect to $\mathrm{L}$ and $\mathrm{H}$. And figure 5 shows the response surface of $\eta$ with respect to Ue and Uc.

The overall performance factor increases with the increase of $\mathrm{H}$ and $\mathrm{L}$, and there is interaction between them. when $\mathrm{H}$ is larger, $\mathrm{L}$ has greater influence on the overall performance factor; the larger $\mathrm{L}$ is, the greater the influence of $\mathrm{H}$ on the overall performance factor. The overall performance factor decreases with the increase of 
Ue and Uc, and the influence of them is small.

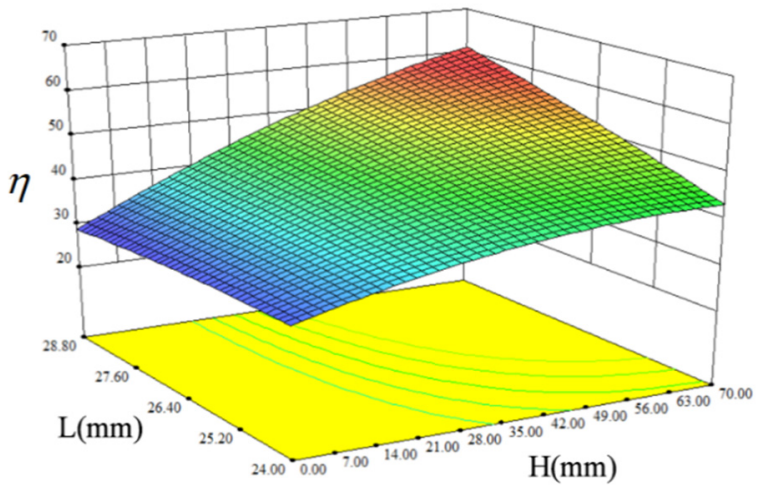

Fig. 4 .Response surface of $\eta$ with respect to $L$ and $H$.

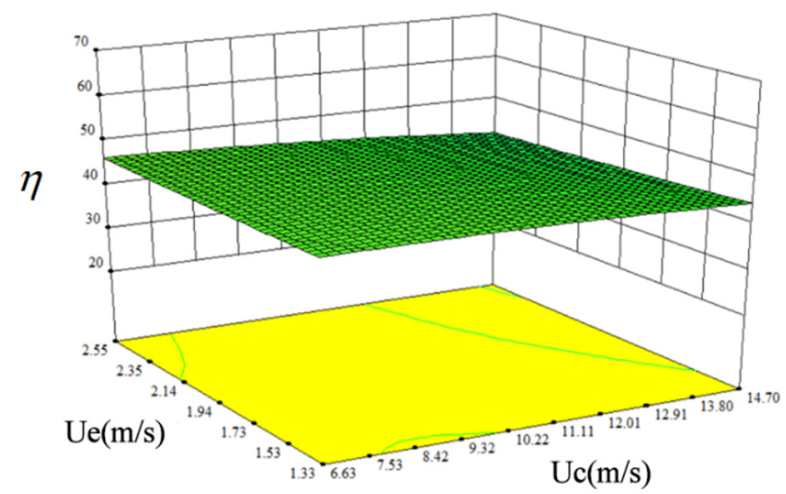

Fig. 5 Response surface of $\eta$ with respect to Ue and Uc.

\subsubsection{Optimization design of $H P H X$}

In this paper, the optimization design of HPHX takes two steps: the first step is to use genetic algorithm to search the largest value of overall performance factor on the response surface model, and the second step is to use numerical calculation method to verify it. The results are listed in Table 3. The error between the largest value of overall performance factor in $\operatorname{RSM}\left(\eta_{1}\right)$ and the numerical result $\left(\eta_{\mathrm{n}}\right)$ is $2.25 \%$, which is in good agreement. The optimal values of $\mathrm{L}, \mathrm{H}, \mathrm{Ue}, \mathrm{Uc}$ are $28.78 \mathrm{~mm}, 66.67 \mathrm{~mm}, 1.4134 \mathrm{~m} / \mathrm{s}$, $12.919 \mathrm{~m} / \mathrm{s}$

Table 3. Optimization result and verification.

\begin{tabular}{|c|c|c|c|c|c|c|}
\hline & $\mathrm{L}$ & $\mathrm{H}$ & $\mathrm{Ue}$ & $\mathrm{Uc}$ & $\eta_{\mathrm{l}}$ & $\eta_{\mathrm{n}}$ \\
\hline Value & 28.78 & 66.67 & 1.4134 & 12.919 & 59.66 & 58.34 \\
\hline
\end{tabular}

\section{Conclusions}

In this paper, optimization design of HPHX using RSM is performed, the overall performance factor is taken as the optimization goal. The following conclusions are summarized.

(1) The increase of $\mathrm{H}$ and $\mathrm{L}$ is beneficial to improve the overall performance of the HPHX; The increase of Ue and $\mathrm{Uc}$ is not conducive to improving the overall performance of the HPHX. Among them, $\mathrm{H}$ and $\mathrm{L}$ has a significant impact, and Ue and Uc has a small impact.

(2) The maximum value of $\eta$ on the response surface is obtained by genetic algorithm, and the optimal values of L, H, Ue, Uc are $28.78 \mathrm{~mm}, 66.67 \mathrm{~mm}, 1.4134 \mathrm{~m} / \mathrm{s}$, $12.919 \mathrm{~m} / \mathrm{s}$.

\section{References}

1. Mamourian M, Shirvan K M, Pop I. (2016) Sensitivity analysis for MHD effects and inclination angles on natural convection heat transfer and entropy generation of Al2O3-water nanofluid in square cavity by response surface methodology. International Communications in Heat and Mass Transfer, 79: 4657.

2. Mckay M D, Beckman R J, Conover W J. (1979) A comparison of three methods for selecting values of input variables in the analysis of output from a computer code. Technometrics, 21(2): 239 -245.

3. Chen Z N. (1991) Experiment design and analysis. Shanghai Jiao Tong University Press, Shanghai.

4. Ramos J, Chong A, Jouhara H. (2016) Experimental and numerical investigation of a cross flow air-towater heat pipe-based heat exchanger used in waste heat recovery. International Journal of Heat and Mass Transfer, 102: 1267-1281.

5. Xin G, Yuankun L, Xiaochao X. (2018) Numerical and experimental investigation of the heat exchanger with trapezoidal baffle. International Journal of Heat and Mass Transfer, 127:598-606. 